\title{
Elaboração e aplicação de instrumentos para avaliação da base de dados Scopus
}

Rosa Mesquita

| Mestranda do PPCCOM/UFRGS; bolsista do CNPq

Sônia Brambilla

Mestranda do PPGCOM/UFRGS

Rita do Carmo Laipelt

| Mestranda do PPGCOM/UFRGS

Maria de Fátima Maia

Mestranda do PPGCOM/UFRGS; bolsista da CAPES

Samile Vanz

Doutoranda do PPGCOM/UFRGS; Professora Assistente do Departamento de

Ciências da Informação da UFRGS

Sonia Elisa Caregnato

Professora Adjunta do Departamento de Ciências da Informaç̧ão e PPGCOM/ UFRGSS; Vice-coordenadora do Grupo de Estudos em Comunicação Cientifica

Apresenta a metodologia e os critérios utilizados para a definição de um instrumento de avaliação de bases de dados, verificando a satisfação dos usuários quanto aos aspectos de conteúdo, funcionalidades do sistema e recuperação da informação. Relata a elaboração e aplicação deste instrumento para avaliação da base de dados Scopus, presente no Portal Periódicos CAPES, por parte de bibliotecários e usuários do Sistema de Bibliotecas da Universidade Federal do Rio Grande do Sul. Os resultados foram positivos em relação à satisfação dos usuários, quanto aos aspectos abordados.

Palavras-chave: Ciência da informação; Bases de dados - avaliação; Estudo de usuário; Scopus. 


\section{Introdução}

grande volume de informações produzidas atualmente tem demandado sistemas que armazenem, gerenciem e disponibilizem ao usuário a informação de que ele necessita, de maneira adequada e satisfatória. Como conseqüência do volume das informações produzidas, crescem também os repositórios e sistemas de recuperação de informação. Assim, a proliferação de bases de dados tem preocupado os profissionais ligados à informação, autores, editores, publicadores e bibliotecários, gerando a premente necessidade de sua avaliação.

Constituindo-se como uma das principais fontes de informação acadêmica, ao permitir acesso a grande parte da produção científica mundial, o Portal Periódicos CAPES reúne, em sua interface, um conjunto expressivo de periódicos nacionais e estrangeiros. São mais de 10.000 revistas e mais de 90 bases de dados referenciais que abrangem todas as áreas do conhecimento. Esta ferramenta é viabilizada pela Coordenação de Aperfeiçoamento de Pessoal de Nível Superior - CAPES -, e disponibilizada a instituições de ensino superior e de pesquisa. Na Universidade Federal do Rio Grande do Sul - UFRGS -, o Portal tem ampla divulgação junto aos professores, pesquisadores e alunos através do Sistema de Bibliotecas - SBU. Uma das incumbências do SBU é avaliar bases de dados online, cujos trials são constantemente oferecidos pelas editoras ao Sistema por um espaço de tempo determinado. Esta avaliação é importante e necessária, pois a incorporação de uma base de dados online numa unidade de informação pressupõe considerável investimento. Sob o aspecto da tomada de decisões, é imprescindível averiguar previamente todos os aspectos da base, vista sob a perspectiva maior de um Sistema de Recuperação de Informações - SRI -, que abrange desde o ambiente gráfico, funcionalidades, performance do sistema, até a satisfação do usuário com os resultados obtidos.

Desta forma, firmou-se uma parceria entre os autores deste trabalho e o Sistema de Bibliotecas da UFRGS para desenvolver, com base em modelos apontados na literatura internacional, instrumentos de avaliação de SRIs, os quais foram aplicados na avaliação da base de dados Scopus, atualmente oferecida pelo Portal Periódicos CAPES. Buscou-se, além de avaliar esta base de dados em particular, desenvolver uma metodologia de avaliação que pudesse ser aplicada em diferentes contextos, inclusive pelo próprio Portal.

Em vista disto, apresenta-se a seguir, a metodologia e os critérios utilizados para a definição de instrumentos de avaliação da satisfação do usuário quanto ao conteúdo, funcionalidades e recuperação da informação. É relatado o processo de avaliação da Scopus junto a bibliotecários e usuários do Sistema de Bibliotecas da UFRGS. Por fim, são relatados os resultados obtidos e sugerido um instrumento para avaliação de bases de dados.

\section{Avaliação de bases de dados}

Os sistemas de recuperação da informação tiveram sua origem nas grandes bases de dados desenvolvidas pelas instituições americanas: National Library of Medicine - NLM -, Department of Defense - DOD - e National Aeronautics and Space Administration - NASA - (LANCASTER, 2004). No atual 
contexto, esses sistemas se difundem, como uma conseqüência da explosão da informação. Assim, a necessidade da avaliação tornou-se ainda mais aguda, e alguns autores vêm se dedicando a este tema e desenvolvendo instrumentos que reúnem critérios para avaliação.

Segundo Lancaster (2004), uma base de dados bibliográficos deve ser avaliada pela sua utilidade ao responder as necessidades de informação, de acordo com quatro critérios principais:

a) cobertura, ou seja, o quanto completo é o conteúdo da base de dados em relação a um assunto;

b) recuperação, já que, tanto quanto ter uma boa cobertura de um assunto, é preciso que estes itens sejam passíveis de recuperação através de uma estratégia de busca não muito complexa;

c) previsibilidade, para que um documento seja reconhecido como relevante a partir das informações contidas na base de dados;

d) atualidade, que é uma medida da velocidade com que novas publicações são incluídas na base.

Para Ahmed, McKnight e Oppenheim (2004), os profissionais da informação mantiveram, durante anos, o domínio do manuseio dos sistemas de recuperação da informação devido a várias razões, tais como a complexidade nos comandos das estratégias de pesquisa e o custo dos sistemas. Estes profissionais eram mediadores da pesquisa, situados entre o usuário e o sistema de informação. Com o crescimento da Internet e das ferramentas como a World Wide Web, deu-se a mudança de comportamento do usuário. $\bigcirc$ profissional da informação, antes o intermediário da pesquisa, vem perdendo espaço para o usuário final, que é considerado hoje a peça chave nas avaliações de sistemas de recuperação da informação.

Sendo assim, o usuário tem sido o objeto de estudo na recuperação da informação. A literatura já classifica o usuário em uma escala que vai de novato a experiente, e muitos estudos afirmam que o usuário novato pode apresentar algumas dificuldades, mas consegue realizar buscas e recuperar informação relevante nas bases de dados. Enquanto um profissional da informação, que é um usuário experiente, recupera um número maior de itens relevantes em uma pesquisa, o usuário novato é mais lento e comete mais erros durante a pesquisa.

Existem muitas técnicas para se avaliar o desempenho de SRIs, baseadas no usuário final, quanto à relevância, pertinência ou precisão, ou nas funcionalidades do sistema. A tendência atual tem sido avaliar conteúdo, produtos e serviços com base na satisfação das necessidades do usuário e nas suas competências, comportamentos e habilidades para a recuperação das informações (AHMED; McNIGHT; OPPENHEIM, 2004; VAN DER GRAAF, 2004; PETERS, 2000; CHIN; DIEHL; NORMAN, 1988). Outros estudos abordam o monitoramento do uso de bases de dados, utilizando os protocolos de comunicação e análise de logs (ERICSSON; SIMON, 1993). As diferentes metodologias têm suas aplicações, dependendo dos objetivos de cada caso; porém, todas têm vantagens e desvantagens. Robertson e Hancock-Beaulieu (1992, p. 465) apontam que escolher o melhor método requer mais pesquisas: "[ . . . ] o desafio para a próxima década será explorar as múltiplas dimensões e componentes da nova geração de SR/s, experimentando-os através do uso dos diversos modelos de avaliação." Ao mesmo tempo, de acordo com 
Kagolovsky e Moehr (2003), praticamente todos os pesquisadores, mesmo os mais otimistas, sabem que nenhuma técnica pode responder a todas as questões, pois há muitas facetas a se analisar num sistema informatizado. Assim, um formulário construído nessa perspectiva deve responder às seguintes questões (SCHAMBER; BATEMAN, 1996):

a) consistir num número considerável de critérios que possam embasar a avaliação, além de perguntas abertas para obter explanações ou informações adicionais;

b) coletar dados quantitativos analisáveis, como freqüência de uso, rankings e estimativas;

c) ser fácil e rápido de analisar, de preferência eletrônica e seqüencialmente;

d) ser adequado para aplicação em grupos variados de usuários e em qualquer tipo de ambiente;

e) ser adaptável a vários objetivos de pesquisa; deve ser integrável a outros instrumentos de coleta de dados, como os que objetivam o estudo da relevância ou pertinência, dependendo das necessidades e interesses de cada caso.

Diversos métodos já foram desenvolvidos para pesquisas de avaliação de bases de dados, tanto utilizando abordagens quantitativas quanto qualitativas. A metodologia para a avaliação de SRIs, adotada no presente trabalho, não ambiciona dar conta de todas as questões envolvidas na avaliação, mas contribuir para a solução de problemas com os quais se deparam as bibliotecas quando da aquisição de novos sistemas ou manutenção dos já existentes.

\section{Metodologia (materiais e métodos)}

\section{AScopus}

A Scopus é uma base de dados multidisciplinar, produzida pela editora Elsevier desde 2004, com cobertura desde 1960, que contém resumos de 27 milhões de artigos, referências e índices da literatura científica, técnica e médica (ELSEVIER, 2004). Pode incluir citações e links para texto completo de artigos, quando esses estão disponíveis. Segundo a empresa, a base de dados indexa 14.000 títulos de periódicos de 4.000 editoras internacionais, com atualizações diárias. Mais de $60 \%$ dos títulos são de outros países que não os Estados Unidos e 85\% do conteúdo é indexado utilizando os vocabulários controlados para a definição das palavras-chave e/ou descritores. A base pesquisa, simultaneamente, o conteúdo dos artigos indexados em sua própria plataforma, na web (através do Buscador Scirus da Elsevier para páginas com conteúdo científico) e ainda bases de patentes sobre o assunto pesquisado. A base Scopus cobre as seguintes áreas do conhecimento: química, física, matemática, engenharia, ciências da saúde e vida, ciências sociais, psicologia, economia, biologia, agricultura, ciências ambientais e ciências gerais.

Jacso (2005), em recente pesquisa, afirma que a Scopus encontra-se, juntamente com o Google Scholar e a Web of Science, entre as maiores bases de dados multidisciplinares. 


\section{Instrumentos de coleta de dados}

O instrumento para avaliação de bases de dados criado nesta pesquisa baseou-se em estudos encontrados na literatura (CHIN; DIEHL; NORMAN, 1988; AHMED; MCNIGHT; OPPENHEIM, 2004).

Inicialmente foram criados três formulários que, após aplicação, mostraram-se muito extensos, com duplicidade de questões e outras inconsistências. Para facilitar a aplicação e análise agruparam-se as questões em dois formulários: um, para ser aplicado nos bibliotecários, e outro, para os usuários. Estes dois formulários foram validados pelo Núcleo de Assessoria Estatística - NAE - da UFRGS os quais encontram-se em anexo neste trabalho.

As questões no formulário de Avaliação do Sistema pelo Bibliotecário foram agrupadas de acordo com os seguintes aspectos: a) conteúdo, com nove questões; b) funcionalidades do sistema, com onze questões e c) recuperação da informação, com doze questões. Quanto às questões do formulário de Avaliação de Satisfação do Usuário foram agrupadas em: a) conteúdo, com cinco questões; b) funcionalidades do sistema, com sete questões e c) recuperação da informação, com nove questões. No final dos instrumentos existe uma questão aberta, solicitando que os respondentes indiquem aspectos positivos ou negativos que julguem necessário salientar.

Para aferir o grau de satisfação foi utilizada a escala de Osgood de diferenciação semântica, que objetiva medir o significado de um objeto para um indivíduo. Consiste em um par de antônimos (adjetivos ou frases), colocados nas duas extremidades de uma escala, sendo a parte interior preenchida pelo respondente, que escolhe a posição que melhor coincide com sua opinião ou atitude. A escolha do número de alternativas, isto é, de variações entre os dois extremos, deve ser em função do tipo de respondente (quanto maior o nível educacional, mais fácil é de distinguir entre muitas alternativas), do tempo disponível para resposta (quanto maior o tempo, mais alternativas) e do grau de satisfação das análises estatísticas (do ponto de vista estatístico, quanto mais alternativas melhor) (OLIVEIRA, 200I).

Nos instrumentos desenvolvidos, cada questão recebeu seu par de adjetivos antônimos, posicionados de forma que o aspecto negativo ficasse ancorado no lado esquerdo e o positivo no lado direito. Entre os termos, foi arranjada uma escala ascendente, de 0 (à esquerda) a 9 (à direita). Questões não respondidas foram consideradas como: não sabe ou não respondeu e desconsideradas do total, para efeitos de avaliação.

\section{Participantes da pesquisa}

Foram sujeitos da pesquisa os bibliotecários e os usuários finais das bibliotecas. Aos bibliotecários, considerados usuários intermediários pelo conhecimento maior sobre as diversas facetas de um sistema de recuperação de informaç̧ões, foram aplicados os três formulários, para que avaliassem a Scopus quanto a satisfação em relação ao conteúdo, funcionalidades do sistema e recuperação da informação. Aos usuários finais foi aplicado somente o formulário de recuperação da informação. 
Sendo a Scopus uma base de dados multidisciplinar foram convidados para testar os três instrumentos e avaliar a base, 20 bibliotecários das seguintes bibliotecas setoriais da UFRGS: Administração; Agronomia; Biblioteconomia e Comunicação; Biociências; Ciências Econômicas; Ciências Sociais e Humanidades; Educação; Educação Física; Enfermagem; Engenharia; Farmácia; Física; Geociências; Informática; Matemática; Medicina/HCPA; Odontologia; Psicologia; Química e Veterinária. Desses convidados, 16 compareceram. Algumas bibliotecas do SBU não participaram por não terem representatividade de assunto na base como, por exemplo, as que atendem as áreas de Artes e Humanidades.

Os usuários finais foram selecionados pelos próprios bibliotecários com base em sua familiaridade com sistemas de recuperação da informação. Assim, foram entregues 32 formulários aos bibliotecários para serem repassados aos usuários, sendo que 12 desses formulários foram retornados para análise.

\section{Procedimentos da pesquisa}

Na primeira parte da coleta, os bibliotecários foram contatados por telefone e a aplicação dos instrumentos ocorreu em duas sessões de 3 horas, no Laboratório de Informática dos cursos de Biblioteconomia e Arquivologia da UFRGS. No início das sessões foi feita uma breve descrição dos propósitos do estudo e dos procedimentos metodológicos que deveriam ser seguidos.

O estudo utilizou uma combinação de métodos para coleta e análise de dados, que incluiu revisão bibliográfica, tarefas de pesquisa e formulários. Conforme Ahmed, McKnight e Oppenheim (2004), cuja metodologia foi adaptada para este trabalho, todos os participantes receberam três tarefas de pesquisa, a serem aplicadas na Scopus, como segue:

I ${ }^{a}$ busca: encontrar artigos sobre um tópico de pesquisa na sua área, utilizando a busca simples;

$2^{\mathrm{a}}$ busca: encontrar artigos escritos por um autor reconhecido em sua área;

$3^{\text {a }}$ busca: encontrar artigos escritos que citam um trabalho localizado na $2^{\mathrm{a}}$ busca.

Não foram determinados termos específicos, tipo de pesquisa ou uso de operadores booleanos. No entanto, foi solicitado aos participantes que explorassem o sistema, com o objetivo de familiarizá-los com a interface, fazendo uso abrangente de todos os recursos e que, num segundo momento, efetuassem a pesquisa, preenchendo os três formulários de avaliação da Scopus.

Para a segunda parte da coleta, os bibliotecários que compareceram à sessão receberam como tarefa aplicar o formulário de avaliação da informação a dois usuários finais de suas bibliotecas. O usuário final foi instruído para explorar a busca na base antes de responder o instrumento de avaliação. 


\section{Tratamento dos dados}

Os dados quantitativos foram tratados estatisticamente na forma de tabelas e gráficos, com o auxílio do programa Excel ${ }^{\circledR}$ para análise qualitativa posterior. As perguntas abertas foram examinadas descritivamente, através de análise textual, sendo destacados os principais pontos levantados. Ainda, foram observadas e anotadas todas as reações dos respondentes durante a aplicação dos formulários para, ao final do estudo, efetuar-se a avaliação dos instrumentos utilizados.

\section{Análise dos dados}

A análise dos dados contemplou os quesitos mais relevantes de cada tópico da avaliação, uma vez que o instrumento de coleta de dados aplicado era específico para cada um dos três temas (conteúdo, funcionalidades e recuperação da informação). Optou-se por apresentar a moda e a mediana das variáveis estudadas, pois elas melhor representam a dispersão da avaliação da satisfação dos usuários de forma geral.

A seguir, é apresentada a análise dos dados em duas etapas, visto que aos bibliotecários foram aplicados os três formulários e, aos usuários das bibliotecas, apenas o formulário de recuperação da informação.

\section{Primeira etapa: Avaliação da Scopus pelos bibliotecários}

A análise dos dados, em relação aos formulários aplicados aos 20 bibliotecários do SBU apresentou os seguintes resultados:

TABELA I - Avaliação de satisfação da Scopus pelos 20 Bibliotecários

\begin{tabular}{|c|c|c|c|}
\hline Itens (Quesitos) & Moda & N. de notas da Moda & Mediana \\
\hline \multicolumn{4}{|l|}{ I CONTEÚDO DO SISTEMA } \\
\hline I.I Áreas de cobertura & 8,0 & 5 & 7,5 \\
\hline 1.2 Período de cobertura & 7,0 & 5 & 7,0 \\
\hline I.3 Comparação com outras bases de dados & 7,0 & 4 & 7,0 \\
\hline \multicolumn{4}{|l|}{2 FUNCIONALIDADES DO SISTEMA } \\
\hline 2. I Link ao texto completo & 8,0 & 5 & 7,0 \\
\hline 2.2 Manuais & 8,0 & 4 & 8,0 \\
\hline 2.3 Organização das informações na tela & 7,0 & 6 & 7,0 \\
\hline \multicolumn{4}{|l|}{3 RECUPERAÇÃO DA INFORMAÇÃO } \\
\hline 3. I Avaliação geral & 9,0 & 5 & 8,0 \\
\hline 3.2 Facilidade de utilização & 9,0 & 6 & 8,0 \\
\hline 3.3 Pesquisa simples & 8,0 & 6 & 8,0 \\
\hline 3.4 Pesquisa avançada & 8,0 & 10 & 8,0 \\
\hline 3.5 Opções de salvamento dos resultados & 8,0 & 7 & 8,0 \\
\hline 3.6 Resultados da busca & 9,0 & 5 & 8,0 \\
\hline
\end{tabular}

Fonte: Dados da pesquisa 
Conforme a $T A B$. I, nas questões relativas à satisfação quanto ao conteúdo do sistema, destaca-se a área de cobertura, com valor modal 8 (com cinco notas) e mediana 7,5. Os demais quesitos deste tópico tiveram tanto para a moda quanto para a mediana 7.

Nas questões de satisfação com as funcionalidades do sistema, tanto a moda do item 2. I, Link ao texto completo (com cinco notas) quanto do 2.2. Manuais foi 8 (com quatro notas) a mediana do primeiro item foi 7 e a do segundo, 8.

Verifica-se, na avaliação das questões de satisfação quanto à Recuperação da informação, que todas receberam nota 8 para a moda e a mediana. Com exceção dos itens 3. I Avaliação geral, 3.2 Facilidade de utilização e 3.6 Resultados da busca cujo valor modal foi 9. Destaca-se também a questão 3.4 Pesquisa avançada que recebeu dez notas 8 (valor modal).

O GRAF. I, abaixo, apresenta o agrupamento das respostas por faixa de valor. Os quesitos numerados referem-se aos itens apresentados na TAB. I.

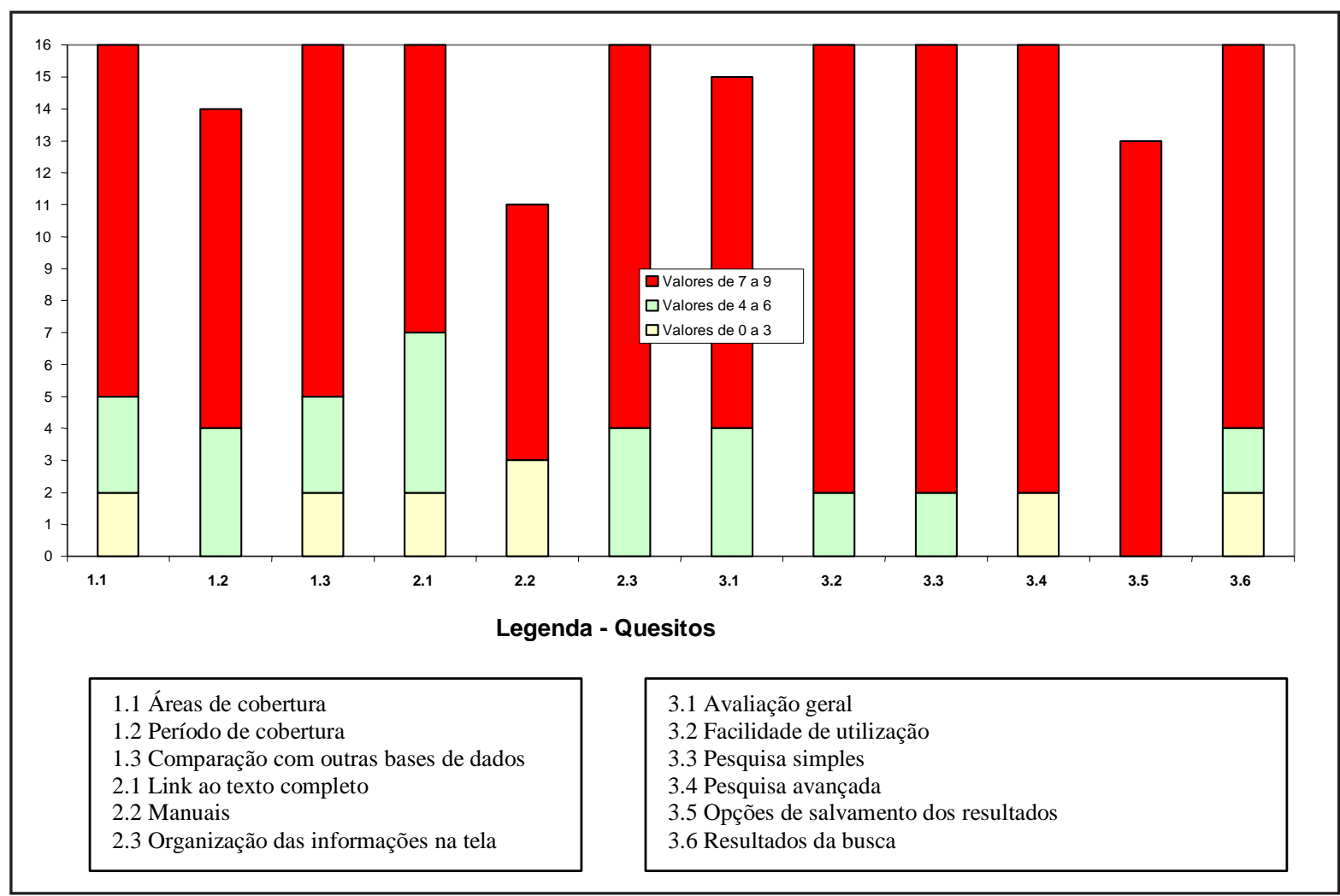

GRÁFICO I - Quantidade acumulada de respostas dos bibliotecários, por faixa de valor Fonte: dados da pesquisa

Na avaliação do conteúdo do sistema, observa-se que, na questão I.I, áreas de cobertura, onze das notas recebidas constam na faixa superior. Destas, nove foram da área de ciências puras, biológicas e exatas e duas das ciências sociais. As cinco notas recebidas nos intervalos inferiores foram das disciplinas da área de ciências sociais. Este resultado reflete a maior cobertura da base Scopus nas áreas das ciências puras, biológicas e exatas. O período de cobertura, questão I.2, recebeu dez notas na faixa superior de 7 a 9 e nenhuma avaliação na menor faixa. $\mathrm{Na}$ comparação com outras bases de dados, quesito 1.3, onze usuários atribuíram notas na faixa de 7 a 9 , três atribuíram notas na faixa 
intermediária e dois na faixa de zero a 3. Observou-se que as maiores notas, na comparação com outras bases de dados, foram atribuídas pelos usuários pertencentes às áreas das ciências puras, biológicas e exatas. Cabe ressaltar que tanto a cobertura quanto a comparação com outras bases de dados receberam avaliações semelhantes por parte dos usuários, demonstrando que há uma relação entre esses dois aspectos.

Quanto à avaliação das funcionalidades do sistema, destaca-se a questão 2.3, organização das informações na tela, que recebeu doze valores na faixa superior e quatro na faixa intermediária, indicando uma tendência de que o sistema propicia aos usuários visualização adequada dos recursos oferecidos. A maioria das respostas das questões 2. I, Link ao texto completo e 2.2, Manuais, estão situadas no intervalo superior. Contudo, em relação à qualidade do suporte técnico oferecido através de manuais, apenas dez dos usuários responderam à questão. $\bigcirc$ alto índice de ausência de respostas pode indicar que os profissionais tiveram dificuldades para responder a questão devido ao tempo exíguo para a análise do conteúdo dos manuais.

Observa-se, na avaliação do tópico recuperação da informação, que a questão 3.I, avaliação geral, recebeu onze notas na faixa superior e quatro na faixa intermediária, revelando que o desempenho geral da base é aceitável. As questões 3.2 facilidade de utilização e 3.3 pesquisa simples foram avaliadas com quatorze notas da faixa superior e duas da faixa intermediária. Destaca-se a questão 3.4 pesquisa avançada que, das quatorze notas recebidas na faixa superior, dez notas foram para o valor modal 8 (conforme TAB. 2) e duas notas na faixa inferior. Também merece destaque a questão 3.5 opções de salvamento dos resultados, que recebeu todas as notas na faixa superior. Pelos resultados obtidos, pode-se considerar que a interface apresentada pela base proporciona um ambiente de fácil navegação para a recuperação das informações.

Os formulários continham uma pergunta aberta encerrando a avaliação, onde foi solicitado aos respondentes que destacassem aspectos positivos e negativos da base. Os bibliotecários citaram problemas com a busca por autor, como segue:

"a busca não respeita a ordem das iniciais dos prenomes, por exemplo, Claudia Lopes Fonseca busca por todos os 'Fonseca' que tenham no prenome C e L, sem levar em conta a ordem de aparecimento";

"o help para pesquisa de autor está diferente do exemplo colocado no guia author search, isto é, as iniciais do prenome estão indicadas com ponto (J.L.) e sem espaço, no help aparecem sem ponto e com espaço";

"em relação às opções de visualização de resultados na Web e patents, estes não foram confiáveis, a busca foi feita por autor e os resultados apresentados nestes links diferiram do nome pesquisado, recuperando todos os sobrenomes e não o nome completo".

Outro aspecto abordado foi quanto à necessidade de utilização de software que permita a configuração do sistema para pessoas portadoras de deficiência visual propiciando a acessibilidade universal. 


\section{Segunda etapa: Avaliação da Scopus pelos usuários das bibliotecas}

A análise dos dados, em relação ao formulário aplicado nos usuários das bibliotecas, apresentou os seguintes resultados:

TABELA 2 - Avaliação de Satisfação da Scopus pelos 12 Usuários das Bibliotecas

\begin{tabular}{l|c|c|c}
\hline Itens (Quesitos) & Moda & N. da Moda & Mediana \\
\hline 3 RECUPERAÇÃO DA INFORMAÇÃO & & & \\
\hline 3.1 Avaliação geral & 8,0 & 7 & 8,0 \\
\hline 3.2 Facilidade de utilização & 9,0 & 5 & 8,0 \\
\hline 3.3 Pesquisa simples & 9,0 & 5 & 8,0 \\
\hline 3.4 Pesquisa avançada & 9,0 & 5 & 8,0 \\
\hline 3.5 Opções de salvamento dos resultados & $7,0,8,0$ e 9,0 & 3 & 8,0 \\
\hline 3.6 Resultados da busca & 9,0 & 5 & 8,0 \\
\hline
\end{tabular}

Fonte: dados da pesquisa

Conforme a TAB. 2, verifica-se que o valor modal do item 3. I avaliação geral foi 8 (com sete notas) e do item 3.5 opções de salvamento dos resultados foi 7, 8 e 9 (cada um com três notas). A moda das demais questões foi 9 (com cinco notas cada). A mediana de todas as questões foi 8. Estes resultados evidenciam a consistência da avaliação positiva da base pelos usuários.

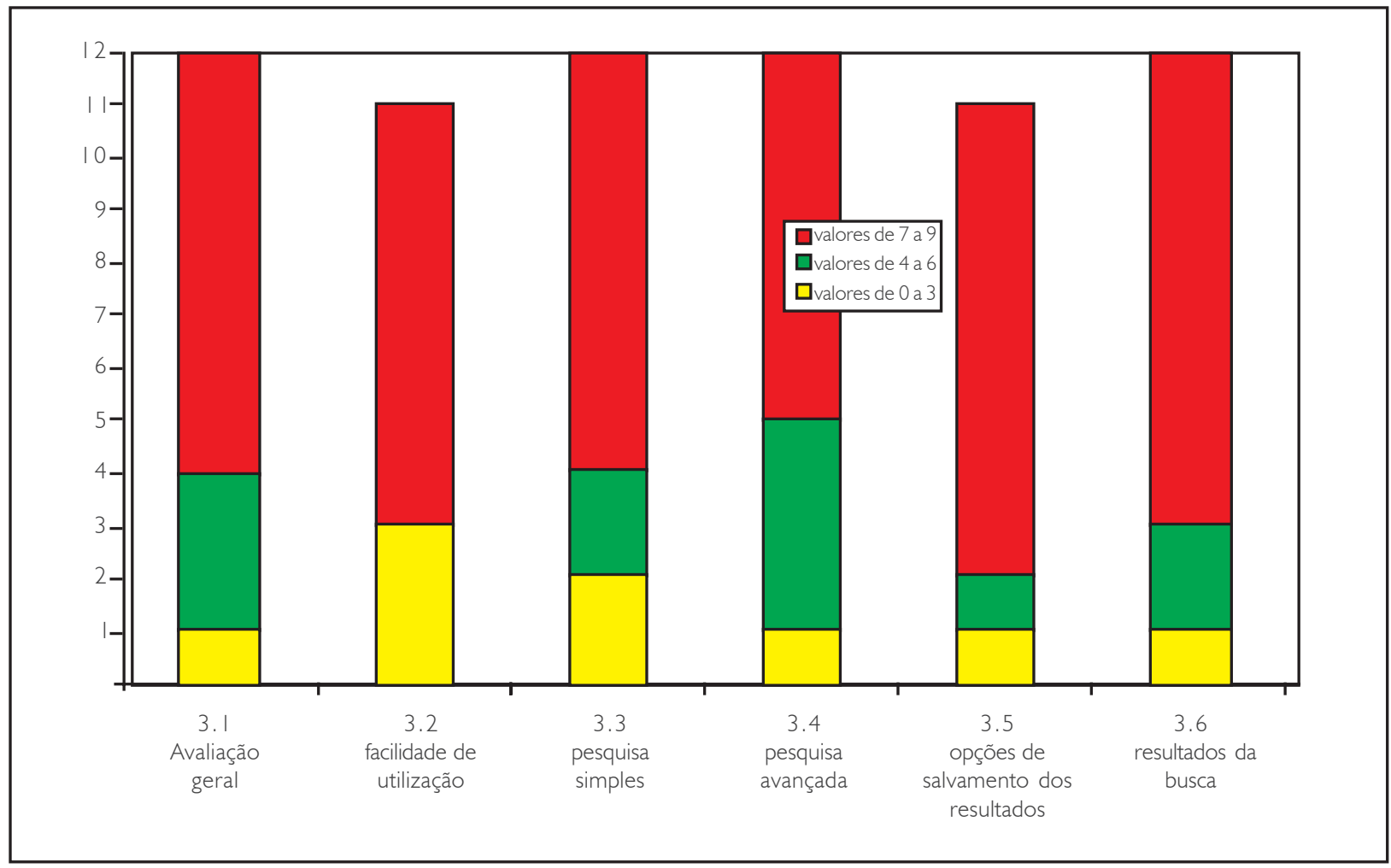

GRÁFICO 2 - Quantidade acumulada de respostas dos usuários das bibliotecas, por faixa de valor Fonte: dados da pesquisa 
O GRAF. 2 apresenta o agrupamento das respostas por faixa de valor. As questões numeradas referem-se aos itens apresentados na TAB. 2. Conforme se observa nesse gráfico, a questão 3. I, avaliação geral, recebeu oito notas na faixa superior (sendo sete notas 8 - valor modal, conforme TAB. 2), três na intermediária e uma na inferior, demonstrando a aprovação dos usuários quanto ao desempenho geral da base. A questão 3.2, facilidade de utilização, teve três respostas na faixa inferior e oito na superior. Observa-se na questão 3.3, pesquisa simples, oito respostas na faixa superior e duas na intermediária e na inferior. Destaca-se a questão 3.5, opções de salvamento dos resultados, que recebeu nove notas na faixa superior e uma nota nas faixas intermediária e inferior e a questão 3.6, resultados da busca que recebeu nove notas na faixa superior, duas na intermediária e uma na inferior.

$\mathrm{Na}$ avaliação dos usuários sobre a base de dados, observa-se que a maioria das notas ficou na faixa superior (7 a 9), demonstrando a apreciação positiva da base no quesito recuperação da informação. Verificou-se que as menores notas foram atribuídas pelos usuários da biblioteca de Educação e Letras, resultado já esperado devido à cobertura da base nestes assuntos. Já entre os de Economia, observaram-se comportamentos distintos, com avaliações negativas e positivas. Percebeu-se que os usuários utilizaram o espaço das questões abertas para reafirmar e/ou justificar as notas que atribuíram à base de dados. Como aspectos positivos, destacaram que a base é acessível, cruza bem dados de diferentes áreas do conhecimento, indexa títulos de periódicos de editoras importantes, é agradável e abrangente. Em relação aos aspectos negativos, mostraram-se insatisfeitos com o fato de terem que fazer um cadastro prévio para obtenção de senha para poder utilizar os recursos de envio de e-mail e que a pesquisa retrospectiva vai somente até 1966.

\section{Considerações finais}

Apesar dos problemas encontrados na busca por autor, a Scopus revelou uma interface de pesquisa amigável, principalmente se comparada a outras bases multidisciplinares. $\bigcirc$ sistema proporciona facilidades na busca e personaliza a visualização dos resultados recuperados, permitindo a adequação destes aos critérios da pesquisa do usuário.

De acordo com as TAB. I e 2, verificou-se que, tanto a moda quanto a mediana de todas as questões, foram iguais ou superiores a 7 e que a dispersão das respostas nos GRAF. I e 2 indicou que a maioria dos respondentes avaliou a base no limite superior da escala para todos os quesitos. Estas considerações nos levam a concluir que a base de dados Scopus foi avaliada positivamente pelos bibliotecários e pelos usuários, quanto à satisfação com o uso, em relação ao conteúdo, às funcionalidades e à interface do sistema (recuperação da informação).

Contudo, o estudo teve como limitação o tempo então disponibilizado pela Editora Elsevier para o trial da base de dados Scopus. Acredita-se que reunir os bibliotecários para a aplicação do instrumento de forma coletiva fez com que muitas informações fossem perdidas, já que não foi possível acompanhar, de maneira individualizada, o uso da base e a reação dos participantes da pesquisa aos formulários aplicados. $O$ processo de avaliação sofreu inúmeras alterações durante sua realização, considerando as reações e 
sugestões dos respondentes da pesquisa. Ao final, resultaram dois formulários, que foram validados pelo NAE/UFRGS e encontram-se em anexo, como sugestão de instrumento para avaliação de bases de dados.

\section{Instruments designing and application for Scopus database evaluation}

The article presents the methodology and criteria used for designing an instrument for database evaluation in order to verify user satisfaction regarding content, system functionality and information retrieval. It also reports an application of such instrument for the evaluation of the Scopus database, on the Portal Periódicos CAPES, by librarians and users from the Library System of the Federal University of Rio Grande do Sul. Results showed that users were satisfied with database features.

Key-words: Information science; Database - evaluation; User studies; Scopus.

\section{Referências}

AHMED, S.IM. Zabed; MeNIIGH, Ciiff; oPpeNHEIM, Charles. A Study of Users' Perfomance and Satisfaction with the Web of Science IR Interface. Journalof Information Science, Cambridge, v. 30, n. 5, p. 459-468, 2004.

CHII, John P:; DIEHL, Virginia A.; NORMAN, KentL. Development of an instrument measuring user satisfaction of the human-computer interface. In: CHr'88: PROCEEDINGS OF THE SICGHI CONFERENCE ON HUMAN FACTORS IN COMPUTING SYSTEMS, 1988, Washington, DC. Proceedings.... New York. ACM. 1988. p. $213-218$.

ElsEVUIER. Scopus. Amsterdam: Elsevier, 2004. Material publicitário.

ERICSSON, A.; SIMON, H.A. Protocol/Analysis. Verbal Reports as Data. Cambridge: MII Press, 1993.

JACSO, P. As we may search - Comparison of major features of the Web of Science, Scopus and Google Scholar citation-based and citation-enhanced databases. Current Science, v. 89, n.9., p. 1537-1547, Nov. 2005.

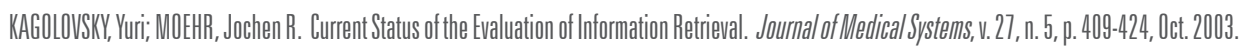

LANCASTER, F.W. Indexą̧ãa e Resumos. teoria e prática. Brasilia: Briquut de Lemos, 2004.

OLIVEIRA, Tânia Modesto Veludo de. Escalas de Mensuraçãão de Atitudes: Thurstone, Osgood, Stapel, Likert, Guttman, Apert. Adminisistraçãa On Line, São Paulo, v. 2, n. 2, abr.jun. 2001. Disponivel em: < http://wwww.fecap.br/adm_online > . Acesso em: 21 maio 2005.

PETERS, Thomas A. Introduction: criteria for assessment of digital library senvices. Library Irends, Illinois, v. 49, n. 2, p.221, Fall 2000.

CAMEY, Susy Alves. Susy Alves Camer. depoimento [11 nov. 2005]. Entrevistadora: Rosa Apel Mesquita. Porto Alegre: UFRGS, 2005.

ROBERTSON, S. E.; HANCCCK-BEAULLE, M. M. On the evaluation of IR systems. Information Processing and Management, Elmsford, v. 28, n. 4, p. 457-466, 1992.

SCHAMBER, Linda; BAFEMAN, Judy. User criteria in relevance evaluation: toward development of a measurement scale. In: ANNUAL MEETING OF THE AMERICAN SOCLETY FOR INFORMATION SCIENCE, 59., Proceedings... [S. I.] : ASIS [s. d.]. v.33, p.218-225.

VAN DER GRAAF, M. A report on the functionality of abstract \& indexing (Affl) database plattorms. 'recent developments, library policies and a new evaluation technique. Amsterdam: Elsevier, 2004. (Scopus White Paper Series, n. 3).

Agradecemos aos bibliotecários do Sistema de Bibliotecas da UFRGS, especialmente a Maria Cristina Bürger e Elenice Ávila da Silva, pela participação nesta pesquisa e ao NAE/UFRGS pela assessoria estatística. 


\section{ANEXOS}

\section{AVALLCÃ̃o DO SISTEMA PELIO BIBLLOTEĆ́rilO}

Solicitamos sua colaboração no sentido de preencher o formulário abaixo com o máximo de fidelidade possível. As informações têm caráter sigiloso e subsidiarão a avaliação de bases de dados.

\section{CONTEÚDO}

I. I Cobertura geográfica

Insatisfeito

$\begin{array}{llllllllll}0 & 1 & 2 & 3 & 4 & 5 & 6 & 7 & 8 & 9\end{array}$

|.2 Período de cobertura

Insatisfeito

Satisfeito

$\begin{array}{llllllllll}0 & 1 & 2 & 3 & 4 & 5 & 6 & 7 & 8 & 9\end{array}$

I.3 Abrangência de áreas do conhecimento Insatisfeito

$\begin{array}{llllllll}2 & 3 & 4 & 5 & 6 & 7 & 8 & 9\end{array}$

1.4 Tipos de documentos indexados (artigos, trabalhos de eventos, teses e dissertações, livros, etc.)

Insatisfeito

$\begin{array}{llllllllll}0 & 1 & 2 & 3 & 4 & 5 & 6 & 7 & 8 & 9\end{array}$

I.5 Importância dos títulos indexados Insatisfeito

$\begin{array}{llllllllll}0 & 1 & 2 & 3 & 4 & 5 & 6 & 7 & 8 & 9\end{array}$

I.6 Completude da cobertura dos títulos (todos os artigos e todos os números da coleção)

Insatisfeito

$\begin{array}{llllllllll}\text { Insatisfeito } & & & & & & & \text { Satisfeito } \\ 0 & 1 & 2 & 3 & 4 & 5 & 6 & 7 & 8 & 9\end{array}$


1.7 Freqüência das atualizações Insatisfeito

$\begin{array}{llllllllll}0 & 1 & 2 & 3 & 4 & 5 & 6 & 7 & 8 & 9\end{array}$

1.8 Qualidade dos resumos Insatisfeito

$\begin{array}{llllllllll}0 & 1 & 2 & 3 & 4 & 5 & 6 & 7 & 8 & 9\end{array}$

1.9 Os resultados das buscas foram:

Insatisfatórios

$\begin{array}{llllllllll}0 & 1 & 2 & 3 & 4 & 5 & 6 & 7 & 8 & 9\end{array}$

\section{FUNCIONALIDADES DO SISTEMA}

2.I Interface gráfica

Desagradável

$\begin{array}{llllllllll}0 & 1 & 2 & 3 & 4 & 5 & 6 & 7 & 8 & 9\end{array}$

2.2 A barra de ferramentas simplifica as tarefas De forma nenhuma

$\begin{array}{llllllllll}0 & 1 & 2 & 3 & 4 & 5 & 6 & 7 & 8 & 9\end{array}$

$2.3 \bigcirc$ sistema mantém você informado sobre o que ele está fazendo? Nunca

Sempre

$\begin{array}{llllllllll}0 & 1 & 2 & 3 & 4 & 5 & 6 & 7 & 8 & 9\end{array}$

2.4 Uso de comandos e ícones

Difícil

Fácil

$\begin{array}{llllllllll}0 & 1 & 2 & 3 & 4 & 5 & 6 & 7 & 8 & 9\end{array}$

2.5 As mensagens de erro:

Nunca ajudam

$\begin{array}{llllllllll}0 & 1 & 2 & 3 & 4 & 5 & 6 & 7 & 8 & 9\end{array}$

$2.6 \bigcirc$ recurso de ajuda (Help/Tutorial)

Nunca auxilia

Sempre auxilia

空

$\begin{array}{llllllllll}0 & 1 & 2 & 3 & 4 & 5 & 6 & 7 & 8 & 9\end{array}$


2.7 Documentação (manuais)

Insatisfeito

$0 \quad 1$

23

4

$\begin{array}{lllll}5 & 6 & 7 & 8 & 9\end{array}$

2.8 Suporte Técnico

Insatisfeito

$0 \quad 1$

23

3

4

5

$6 \quad 7$

Satisfeito

89

2.9 Integração com outros sistemas de recuperação de informações Insatisfeito

$0 \quad$ I

2

3

45

$6 \quad 7$

Satisfeito

89

2. 10 Personalização do sistema

Insatisfeito

$0 \quad$ ।

23

4

5

$6 \quad 7$

Satisfeito

89

2.II Usar o sistema é:

Difícil

$0 \quad 1$

2

3

4

5

$6 \quad 7$

89

\section{ReCUPERAÇÃO DA INFORMAÇÃO}

3.I Pesquisa Simples

Insatisfeito

0

2

3

45

5

$6 \quad 7$

Satisfeito

9

3.2 Pesquisa avançada

Insatisfeito

$0 \quad 1$

23

4

$6 \quad 7$

Satisfeito

9

3.3 Tesauro ou vocabulário controlado

Insatisfeito

$0 \quad 1$

23

3

45

5

$6 \quad 7$

Satisfeito

9

3.4 Qualidade da indexação

Insatisfeito
0
2
3
4
5
$6 \quad 7$
89

Satisfeito 
3.5 Opções de apresentação dos resultados de busca (ordenar por data, idioma, tipo de publicação, área, etc.)

Insatisfeito

$\begin{array}{llllllllll}0 & 1 & 2 & 3 & 4 & 5 & 6 & 7 & 8 & 9\end{array}$

3.6 Refinamento da busca (por data, idioma, tipo de publicação, área, por autor, título, etc.)

Insatisfeito
0
23
$4 \quad 5$

$\begin{array}{lllllllll}0 & 2 & 3 & 4 & 5 & 6 & 7 & 8 & 9\end{array}$

Satisfeito

3.7 Opções de salvamento dos resultados de busca (exportar, enviar por email, imprimir, formatar texto em html, pdf, doc, etc.)

Insatisfeito

Satisfeito

$\begin{array}{llllllllll}0 & 1 & 2 & 3 & 4 & 5 & 6 & 7 & 8 & 9\end{array}$

3.8 Possibilidade de personalização dos serviços (armazenar estratégias e resultados de pesquisas anteriores, envio de alertas por e-mail, envio de alertas de citações)

$\begin{array}{llllllllll}\text { Insatisfeito } & & & & & & & \text { Satisfeito } \\ 0 & 1 & 2 & 3 & 4 & 5 & 6 & 7 & 8 & 9\end{array}$

3.9 Oferece link para artigos na íntegra Insatisfeito

Satisfeito

$\begin{array}{llllllllll}0 & 1 & 2 & 3 & 4 & 5 & 6 & 7 & 8 & 9\end{array}$

3. 10 Apresenta índice de citação (informa quantas vezes os artigos foram citados) Insatisfeito

Satisfeito
0
23
45
$6 \quad 7$
89

3. I I Recursos de busca fonética (quando um termo é digitado incorretamente o sistema oferece opções) Insatisfeito

Satisfeito

$\begin{array}{llllllllll}0 & 1 & 2 & 3 & 4 & 5 & 6 & 7 & 8 & 9\end{array}$

3.12 Percepção geral em relação à Base de Dados Insatisfeito

$\begin{array}{llllllllll}0 & 1 & 2 & 3 & 4 & 5 & 6 & 7 & 8 & 9\end{array}$




\section{CITE OS ASPECTOS POSIIIUOS OU NEGATIUOS SOBRE A BASE DE DADOS QUE JULGAS NECESSÁRIO SALIENTAR.}

\section{AVALIAÇÃO DE SATISFAÇÃO DO USUÁRIO}

Solicitamos sua colaboração no sentido de preencher o formulário abaixo com o máximo de fidelidade possível. As informações têm caráter sigiloso e subsidiarão a avaliação de bases de dados.

\section{CONTEÚDO}

I. I Cobertura geográfica

Insatisfeito

$\begin{array}{llllllllll}0 & 1 & 2 & 3 & 4 & 5 & 6 & 7 & 8 & 9\end{array}$

1.2 Período de cobertura dos resultados Insatisfatório

Satisfatório

$\begin{array}{llllllllll}0 & 1 & 2 & 3 & 4 & 5 & 6 & 7 & 8 & 9\end{array}$

1.3 Tipos de documentos indexados (artigos, trabalhos de eventos, teses e dissertações, livros, etc.)

Insatisfeito

Satisfeito

$\begin{array}{llllllllll}0 & 1 & 2 & 3 & 4 & 5 & 6 & 7 & 8 & 9\end{array}$

I.4 Importância dos títulos indexados Insatisfeito

Satisfeito

$\begin{array}{llllllllll}0 & 1 & 2 & 3 & 4 & 5 & 6 & 7 & 8 & 9\end{array}$

I.5 Os resultados das buscas foram: Insatisfatórios

Satisfatórios

$\begin{array}{llllllllll}0 & \mathrm{I} & 2 & 3 & 4 & 5 & 6 & 7 & 8 & 9\end{array}$

\section{FUNCIONALIDADES DO SISTEMA}

2. I Interface gráfica

\begin{tabular}{lllllllll} 
Desagradável & & & & & & & \multicolumn{2}{c}{$\begin{array}{c}\text { Agradável } \\
0\end{array}$} \\
\hline & 2 & 3 & 4 & 5 & 6 & 7 & 8 & 9
\end{tabular}

2.2 A barra de ferramentas simplifica as tarefas

De forma nenhuma

Totalmente

$\begin{array}{llllllllll}0 & 1 & 2 & 3 & 4 & 5 & 6 & 7 & 8 & 9\end{array}$


3.5 Opções de salvamento dos resultados de busca (exportar, enviar por email, imprimir, formatar texto em html, pdf, doc, etc.)

Insatisfeito

Satisfeito

$\begin{array}{llllllllll}0 & 1 & 2 & 3 & 4 & 5 & 6 & 7 & 8 & 9\end{array}$

3.6 Possibilidade de personalização dos serviços (armazenar estratégias e resultados de pesquisas anteriores, envio de alertas por e-mail, envio de alertas de citações)

Insatisfeito

$\begin{array}{llllllllll}0 & 1 & 2 & 3 & 4 & 5 & 6 & 7 & 8 & 9\end{array}$

3.7 Oferece link para artigos na íntegra

Insatisfeito

$\begin{array}{llllllllll}0 & 1 & 2 & 3 & 4 & 5 & 6 & 7 & 8 & 9\end{array}$

3.8 Apresenta índice de citação (informa quantas vezes os artigos foram citados)

Insatisfeito

Satisfeito

$\begin{array}{llllllllll}0 & 1 & 2 & 3 & 4 & 5 & 6 & 7 & 8 & 9\end{array}$

3.9 Percepção geral em relação à Base de Dados

Insatisfeito

$\begin{array}{llllllllll}0 & 1 & 2 & 3 & 4 & 5 & 6 & 7 & 8 & 9\end{array}$

\section{CITE OS ASPECTOS POSITIUOS OU NEGATIVOS SOBRE A BASE DE DADOS QUE JULGAS \\ NECESSÁRIO SALIENTAR.}

